\title{
Blunt head injury resulting in formation of the superficial temporal artery aneurysm
}

\author{
Jakub Kaczynski \\ Department of Vascular Surgery, ABM University Health Board, Swansea, UK
}

Correspondence to Jakub Kaczynski, jakub.kaczynski@hotmail.co.uk

\section{DESCRIPTION}

A 30-year-old healthy man presented with a 2-week history of a painful lump over the left temple. The lump expanded when the patient coughed or exercised in the gym. Symptoms developed after the patient sustained a blunt trauma (direct blow with a glass bottle) to the left temporal region.

On examination, a $1 \mathrm{~cm}$ hemispherical and tender bleb was found over the left temple (figure 1). The lump was pulsatile and disappeared on the compression of the ipsilateral superficial temporal artery (STA). The remainder of the abdominal and cardiovascular examination was normal. A hand-held Doppler confirmed the presence of blood flow within the lump. Subsequently, a Duplex ultrasound scan was arranged and confirmed the diagnosis

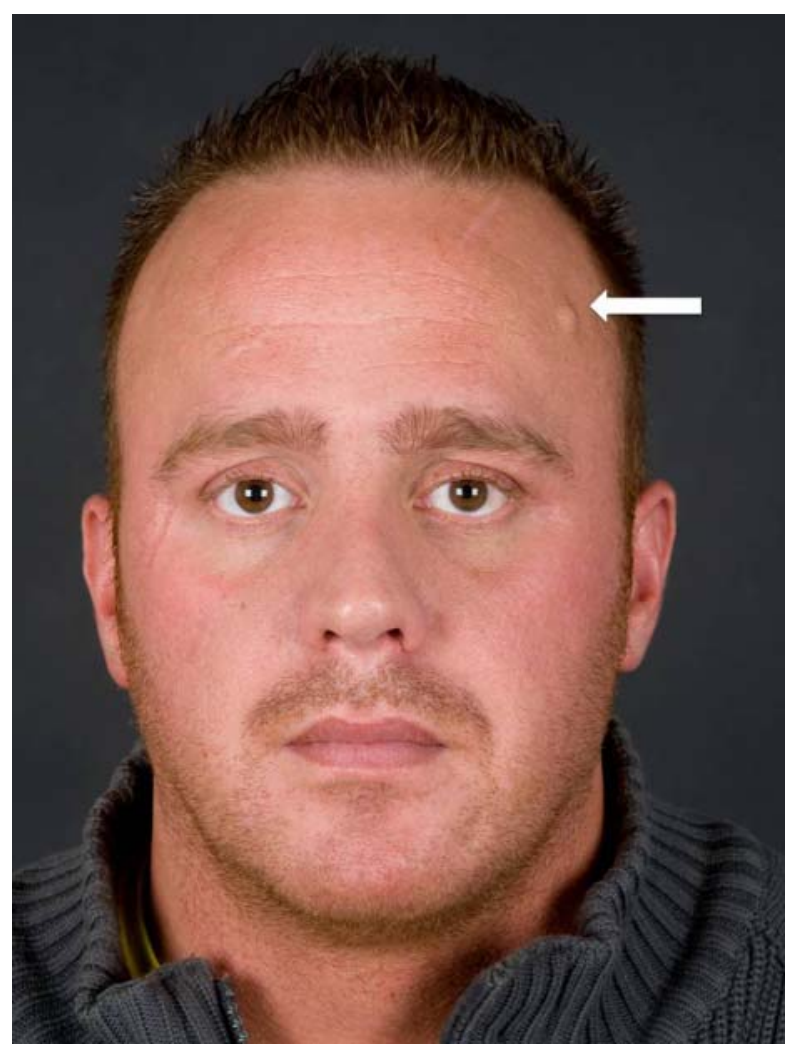

Figure 1 Traumatic true aneurysm in the superficial temporal artery. of the STA true aneurysm with a $3.9 \mathrm{~mm}$ anterior-posterior diameter (figures 2 and 3 ).

STA aneurysms (true and false), in more than two-thirds of cases, are caused by a blunt trauma. ${ }^{1}$ They predominantly affect young men and usually develop 2-6 weeks after the initial injury. In the majority of cases (95\%), this leads to the development of the pseudoaneurysm rather than true STA aneurysm. ${ }^{1} 2$

In true aneurysm, the vessel wall usually is contused and weakened. ${ }^{3}$ It results in visible and palpable pulsation as a consequence of a progressive dilatation of the damaged arterial wall. ${ }^{13}$ However, pulsation may be absent owing to complete thrombosis of the aneurysmal sac. ${ }^{4} 5$ Therefore, the STA aneurysms can easily be mistaken for a sebaceous cyst, lipoma, lymphadenopathy, arteriovenous fistula, haematoma or an abscess. ${ }^{5}$ Misdiagnosis can have devastating consequences including massive haemorrhage after needle aspiration ${ }^{3}$

The pseudoaneurysm is characterised by complete or incomplete arterial wall disruption. ${ }^{1}{ }^{3}$ In such circumstances, extravasation of the blood forms the haematoma with a surrounding fibrous pseudocapsule. ${ }^{13}$ Subsequent lysis of the intraluminal thrombus recanalises the vessel and leads to ongoing aneurysm expansion.

Regardless of the histopathological picture, diagnosis is established on the basis of the history and examination supported by diagnostic evaluation. The most accurate and non-invasive imaging modality is a duplex ultrasound. Other imaging techniques such as CT with contrast and magnetic resonance angiography are useful in cases where intracerebral aneurysms are suspected. ${ }^{5}$

Angiography should be reserved for diagnosis of difficult cases, but at the same time provides an opportunity to perform endovascular treatment.

Treatment options in true and pseudoaneurysms are similar and vary, including ultrasound compression, conservative (wait and watch) treatment, surgical excision with ligation or endovascular obliteration. ${ }^{5} 6$ Each treatment option is associated with risks and requires careful consideration. The main purpose is to reduce the risk of the haemorrhage and improve cosmetic results. Although the natural history of true STA aneurysms remains unclear, the false ones tend to enlarge, thrombose or rupture. ${ }^{2}$

Ultrasound-guided compression for about $15 \mathrm{~min}$ aims at obliteration of the aneurysm by causing thrombus formation. ${ }^{2} 6$ This technique can be used to treat small aneurysms but often fails. ${ }^{1} 6$ 


\section{BMJ Case Reports}

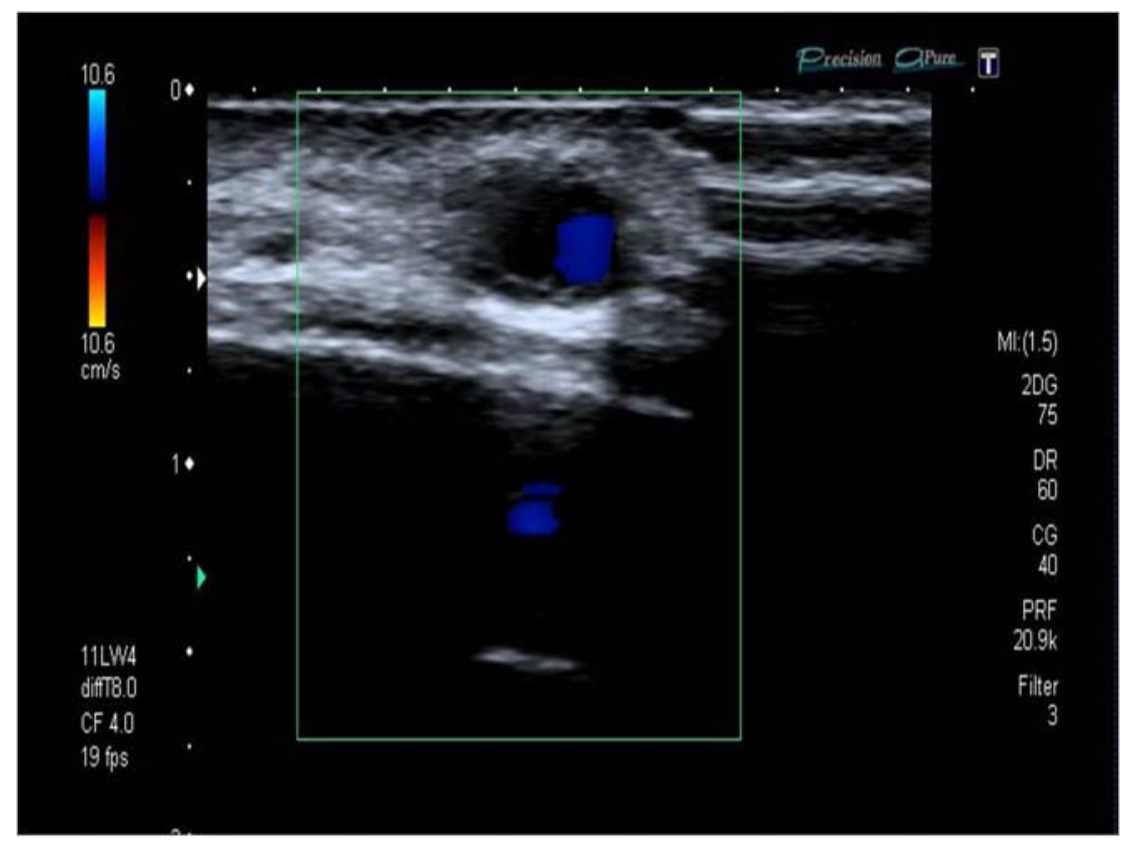

Figure 2 Cross-sectional view demonstrating thrombus and flow (blue colour) within the aneurysm.

The endovascular techniques offer a promising alternative and several case reports have described successful use of embolisation by thrombin or coils. ${ }^{6}$

Direct thrombin injection is a simple method, but can lead to complications such as an allergic reaction, risk of recanalisation, intravascular thrombosis, scalp necrosis and a distal ischaemia. ${ }^{6-8}$ The most serious risk is that of an ischaemic stroke due to migration of the injected particles to the brain. Therefore, coil embolisation is another tool with no reported allergic reactions and a significantly reduced risk of cerebrovascular event. ${ }^{79}$ The literature also reports successful use of the N-butyl-2-cyanoacrylate plus isophendylate, polyvinyl alcohol or gelatine sponge in embolisation. 36
Despite the advance in the minimally invasive techniques, standard surgery remains the gold standard treatment and includes the excision of the lesion (aneurysm) by ligation of the proximal and distal ends of the STA. It is a simple and effective procedure performed under local anaesthesia with no reported recurrences and minimal scarring. ${ }^{2}$ The potential risks include the facial nerve damage in aneurysms located close to the parotid gland. ${ }^{7}$

In this case, following discussion of all the potential risks and benefits with the patient, a standard surgical approach was recommended as the most appropriate treatment option. However, the patient declined any intervention and subsequently did not attend the outpatient follow-up. Therefore, the clinical outcome is unknown.

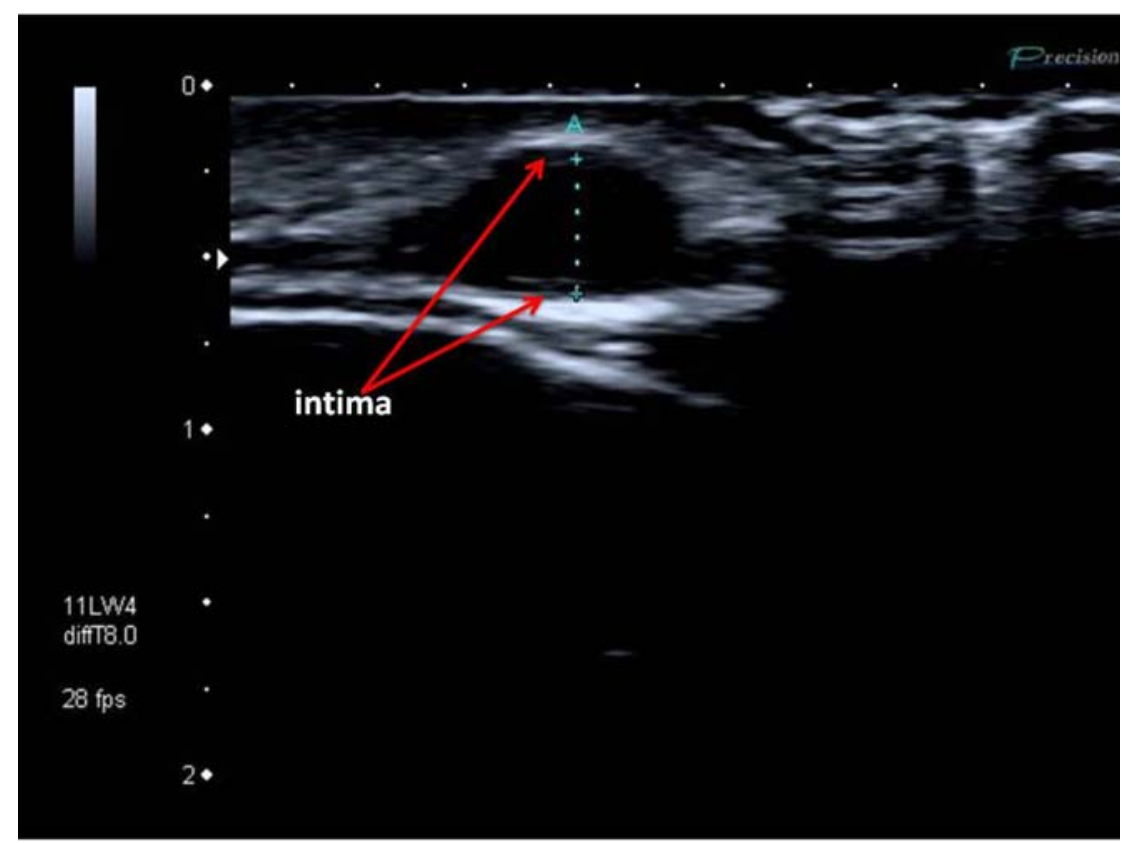

Figure 3 Longitudinal view confirming true aneurysm by the continuity of the intima. 


\section{Learning points}

- Medical history and findings of examinations are vital in establishing the correct diagnosis of STA aneurysms.

- Any patients presenting with a 'lump' after head trauma should be considered for the possibility of an STA aneurysm.

- A hand-held Doppler is an easy bedside test to evaluate a 'lump' for vascular component.

- A duplex ultrasound scan remains the most accurate non-invasive imaging modality prior to any form of intervention such as aspiration or surgical exploration.

- Surgery is the treatment of choice.

\section{REFERENCES}

1. Fox JT, Cordts PR, Gwinn BC II. Traumatic aneurysm of the superficial temporal artery: case report. J Trauma 1994;36:562-4.

2. Silverberg D, Teodorescu V. True aneurysm of the superficial temporal artery. EJVES Extra 2005;9:126-8.

3. Buchanan C, Reeder C. Traumatic pseudoaneurysm of the superficial temporal artery: two cases. J Am Osteopath Assoc 2001;101:284-7.

4. Bailey IC, Kiryabwire JW. Traumatic aneurysms of the superficial temporal artery. Br J Surg 1973;60:530-2.

5. Gull S, Badawy A, Chaudhuri A. The 'pulsatile' sebaceous cyst: beware of a superficial temporal artery aneurysm. BMJ Case Rep 2009; 10.1136/bcr.03. 2009.1698, Published 26 May.

6. Yang HJ, Choi YH. Posttraumatic pseudoaneurysm in scalp treated by direct puncture embolisation using N-Butyl-2-Cyanoacrylate: a case report. Korean J Radiol 2005;6:37-40.

7. Hong JT, Lee SW, Ihn YK, et al. Traumatic pseudoaneurysm of the superficial temporal artery treated by endovascular coil embolisation. Surg Neurol 2006;66:86-8

8. Komiyama M, Nakajima H, Nishikawa M, et al. Endovascular treatment of traumatic aneurysms of the superficial temporal artery. J Trauma 1997:43:545-8.

9. Sanchez F, Delgado F, Ramos M. Pseudoaneurysm of the superficial temporal artery treated by embolisation: report of a case. J Oral Maxillofac Surg 2000; 58:819-21.
Competing interests None.

Patient consent Obtained.

This pdf has been created automatically from the final edited text and images.

Copyright 2012 BMJ Publishing Group. All rights reserved. For permission to reuse any of this content visit

http://group.bmj.com/group/rights-licensing/permissions.

BMJ Case Report Fellows may re-use this article for personal use and teaching without any further permission

Please cite this article as follows (you will need to access the article online to obtain the date of publication).

Kaczynski J. Blunt head injury resulting in formation of the superficial temporal artery aneurysm. BMJ Case Reports 2012;10.1136/bcr-02-2012-5818, Published XXX

Become a Fellow of BMJ Case Reports today and you can:

- Submit as many cases as you like

- Enjoy fast sympathetic peer review and rapid publication of accepted articles

- Access all the published articles

- Re-use any of the published material for personal use and teaching without further permission

For information on Institutional Fellowships contact consortiasales@bmjgroup.com

Visit casereports.bmj.com for more articles like this and to become a Fellow 